\title{
STRAIN RATE DEPENDENT MICROPLANE CONSTITUTIVE MODEL FOR COMMINUTION OF CONCRETE UNDER PROJECTILE IMPACT
}

\author{
KEDAR S. KIRANE*, YEWANG SU*† AND ZDENĚK P. BAŽANT*๋ \\ *Northwestern University \\ Evanston, IL USA \\ e-mail: kedarkirane2011@u.northwestern.edu
}

${ }^{\dagger}$ State Key Laboratory of Nonlinear Mechanics, Institute of Mechanics, Chinese Academy of Sciences Beijing, China

e-mail: yewangsu@imech.ac.cn

${ }^{\ddagger}$ Northwestern University

Evanston, IL USA

e-mail: z-bazant@northwestern.edu

Key words: Comminution, Microplane model, Concrete, Dynamic fracture

\begin{abstract}
The pulverization, fracturing and crushing of materials, briefly called comminution, creates numerous cracks which dissipate a large amount of kinetic energy during projectile impact. At high shear strain rates $\left(10 / \mathrm{s}-10^{6} / \mathrm{s}\right)$, this causes an apparent large increase of strength, called 'dynamic overstress'. This long debated phenomenon has recently been explained by the theory of release of local kinetic energy of shear strain rate in finite size particles that are about to form. The theory yields the particle size and the additional kinetic energy density that must be dissipated in finite element codes. In previous research, it was dissipated by additional viscosity, in a model partly analogous to turbulence theory. Here it is dissipated by scaling up the material strength. Microplane model M7 is used and its stress-strain boundaries are scaled up by theoretically derived factors proportional to the $-4 / 3^{r d}$ power of the effective deviatoric strain rate and to its time derivative. The crack band model with a random tetrahedral mesh is used and all the artificial damping is eliminated from the finite element program. The scaled model M7 is seen to predict the crater shapes and exit velocities of projectiles penetrating concrete walls as closely as the previous models. The choice of the finite strain threshold for element deletion, which can have a big effect, is also studied. It is proposed to use the highest threshold above which a further increase has a negligible effect.
\end{abstract}

\section{INTRODUCTION}

The dynamic comminution (i.e., fragmentation, pulverization and crushing) of materials is of interest for many practical purposes, such as explosion effects on concrete structures, impact of metals, composites and ceramics, rock blasting and fracturing of gas or oil shale by chemical explosions or electro-hydraulic pulsed arc in a horizontal borehole [1, 2, 2, -5, 5,-17, 17,-19, 19.
31]. This article deals with the projectile impact onto concrete walls.

The key aspect to be captured in predictive models for comminution is the so-called 'dynamic overstress', a physical phenomenon manifested at strain rates higher than $1 / \mathrm{s}$. Due to this effect, the material strength needed to fit data on projectile penetration increases enormously compared to that predicted by standard 
strain rate effects (i.e. activation energy controlled bond breakage at crack tips and viscoelasticity of the material between the cracks). In [23, 24], a physical justification of this dynamic overstress was provided by considering the fracturing of concrete to be driven not by the release of strain energy, as in classical fracture mechanics, but by the release of kinetic energy of shear strain rate field with forming particles as the concrete is getting comminuted into small fragments. This new theory, which bears some analogy to turbulence, was shown to give a strain-rate dependent expression for the additional density $\Delta \mathcal{K}$ of kinetic energy that drives the comminution and must be dissipated in the finite element code. There are various methods to dissipate it, and apparently it does not matter which one is adopted.

Some investigators have dealt with this problem by adjusting the strain-dependent strength limits (or boundaries) of the damage model e.g., in [7] for the microplane model M4. The adjustment was done so as to fit the exit velocities of projectile penetrating a wall. However, lacking physical justification, such an adjustment can hamper predictive capability in other loading scenarios, quasi-static or dynamic. Here, the method of adjusting the boundaries is applied to the microplane model M7 with an important improvement that the boundaries are raised such that the additional energy dissipated equals the theoretically calculated additional kinetic density $\Delta \mathcal{K}$ of the shear strain rate field in the forming particles. This method leads to a strength increase depending on both the first and second time derivatives of the deviatoric strain. It is shown to fit well the exit velocities of missiles penetrating concrete slabs. What is important is that this is achieved without a loss of predictive capability in other dynamic, quasi-static and multi-axial loading scenarios. The present theory can be found in detail in [27] and here it is presented in abbreviated form.

\section{OVERVIEW OF THE KINETIC THE- ORY OF COMMINUTION}

The physical source of 'dynamic overstress' during impact was traced to the dissipation of the local kinetic energy of shear strain rate within finite size comminuting particles [23]. Let $\epsilon_{D i j}$ denote the deviatoric strain tensor and the superior dot the derivatives with respect to time t. Then, the density of kinetic energy of effective deviatoric strain rate $\dot{\epsilon}_{D}=\sqrt{\dot{\epsilon}_{D i j} \dot{\epsilon}_{D i j} / 2}$ is dissipated by creating interface fractures resulting in many particles.

Consider an idealized dynamic fracture process in which the solid is comminuted to identical prismatic particles of length $h$ and hexagonal cross section of side $h / 2$, at a deviatoric strain rate $\dot{\epsilon}_{D}$ (Fig. 1). Analysis of the kinematics and comparison of the kinetic energy of particles before (Fig. 1 b) and after (Fig. 14) the interface fracture showed [23] that, for a motion in the plane of maximum shear strain, the local kinetic energy of the particles that are about to form, per unit volume of material, $\Delta \mathcal{K}$, is additive to, and separable from, the global kinetic energy.

The global kinetic energy corresponds to the motion of the centers of the particles whose formation is imminent. For a given $\dot{\epsilon}_{D}$, the drop in kinetic energy per unit volume is found to be [23]:

$$
-\Delta \mathcal{K}=c_{k} \rho h^{2} \dot{\epsilon}_{D}^{2}
$$

where $c_{k}=I_{p} /\left(2 h V_{p}\right), \rho=$ mass density, $V_{p}=3 \sqrt{3} h^{3} / 8$ and $I_{p}=5 \sqrt{3} h^{4} / 128=$ volume and polar moment of inertia of each hexagonal prism about its axis, respectively.

In reality, the size of comminuted particles is never uniform but varies randomly within a certain range, $s \in(h, H)$ where $h, H=$ minimum and maximum sizes, and $s=$ variable particle size. Schuhmann's empirical power law [33] is adopted to describe the cumulative distribution. It gives the volume fraction of particles of sizes between $h$ to $s$ :

$$
F(s)=\frac{s^{k}-h^{k}}{H^{k}-h^{k}}, \quad s \in(h, H), F(s) \in(0,1)
$$


where $(k \approx 0.5)=$ empirical constant [23].

Then, introducing the simplifying hypothesis that the kinetic energy drop for variable particle size can be obtained by summing the energy losses for all infinitesimal intervals $(s, s+$ $\mathrm{d} s$ ) calculated for each interval as if the particle size and shape were uniform. Integration furnishes the kinetic energy drop for particles of all sizes per unit volume [23]:

$$
-\Delta \mathcal{K}=\int_{s=h}^{H} c_{k} \rho s^{2} \dot{\epsilon}_{D}^{2} \mathrm{~d} F(s)=C_{k} \rho h^{2} \dot{\epsilon}_{D}^{2}
$$

where $C_{k}=\frac{k}{k+2} \frac{(H / h)^{k+2}-1}{(H / h)^{k}-1} c_{k}$. The interface area of uniform particles of size $s$ per unit volume of material is $c_{s} / s$ where $c_{s}=$ dimensionless constant. For particle size distributed according to Eq. (2), the particle interface area per unit volume of material is obtained as [23]:

$$
\begin{aligned}
S & =\int_{s=h}^{H} \frac{c_{s}}{s} \mathrm{~d} F(s)=\frac{C_{s}}{h} \\
\text { where } \quad C_{s} & =\frac{c_{s} k}{k-1} \frac{(H / h)^{k-1}-1}{(H / h)^{k}-1}
\end{aligned}
$$

is a dimensionless constant [23]. Eq. (4) adds, or integrates, the energy dissipations for different particle sizes and is calculated under the assumption that for each size there is a regular array of equal-size particles.

Then, considering the dynamic fracture criterion [23, 25] to be $-\Delta \mathcal{K}=S \Gamma$, an overall energy balance is imposed (where $\Gamma=$ interface fracture energy of the comminuting particles). Substitution of eqs 3 and 4 gives the minimum particle size as

$$
h=C_{r}\left(\frac{\Gamma}{\rho \dot{\epsilon}_{D}^{2}}\right)^{1 / 3}
$$

where $\quad C_{r}=\left(\frac{c_{s}}{c_{k}} \frac{k+2}{k-1} \frac{(H / h)^{k-1}-1}{(H / h)^{k+2}-1}\right)^{1 / 3}$

where $C_{r}$ is a dimensionless constant. As seen in Eq. (7), the particle size is proportional to the $-2 / 3$ power of the effective deviatoric strain rate. A higher effective deviatoric strain rate gives a greater kinetic energy release, which leads to smaller particles, and thus to a greater interface area and more dissipation.

In [23], Eq. 7] was used as an explicit formula in which the value of $H / h$ was estimated. It should be noted that this estimate might have some errors. An alternate approach could be using a variable $H / h$ where $H$ is constant but $h$ is not. But this would require a complete rederivation of the theory since it cannot be used in arriving at Eq. (6). Such a theory might be more complicated without a very significant improvement in predictions. Hence, we assume a constant $H / h$.

Then, substitution of Eq. (6) into Eq. (3) gives the kinetic energy drop in terms of only the effective deviatoric strain rate and the material parameters:

$$
-\Delta \mathcal{K}=A \dot{\epsilon}_{D}^{2 / 3}
$$

where $A=\left(C_{0} \Gamma^{2} \rho\right)^{1 / 3}$ and $C_{0}=C_{k}^{3} C_{r}^{2}$ is a dimensionless constant. Note that assuming a hexagonal shape of particles is not a strict requirement of the formulation. In reality the shapes of the particles are surely to be variable, but the basic functional form of the equations is not expected to change.

Furthermore, note that in an actual impact event, $\dot{\epsilon}_{D}$ need not be constant during comminution of a given material volume. So it is useful to obtain the drop in kinetic energy in one time increment of the comminution process. Accordingly, as proposed in [25] the increment of drop in kinetic energy is given by

$$
\mathrm{d}(-\Delta \mathcal{K})=\frac{2 A}{3} \dot{\epsilon}_{D}^{-1 / 3} \mathrm{~d} \dot{\epsilon}_{D}
$$

Thus, in a given strain increment, the drop in kinetic energy depends not only on the strain rate, but also its increment. Any scaling of the boundaries of a constitutive model to account for comminution, must be consistent with this dependence. Such a formulation is proposed in the next section, for the microplane model M7.

\section{MICROPLANE MODEL M7 WITH SCALED BOUNDARIES}

The microplane model M7 is the latest version in a series of progressively improved mi- 
croplane models developed first for concrete and then extended to other quasibrittle materials. The microplane model, supplemented by a suitable localization limiter with material characteristic length, has been proven to give rather realistic predictions of the constitutive and damage behavior of quasi-brittle materials over a broad range of loading scenarios, including uniaxial, biaxial and triaxial loadings with post-peak softening, compression-tension load cycles, opening and mixed mode fractures, tension-shear failure and axial compression followed by torsion [34, 35].

The basic idea of the microplane model is to express the constitutive law not in terms of tensors, but in terms of the vectors of stress and strain acting on a generic plane of any orientation in the material microstructure, called the microplane. The use of vectors is analogous to the Taylor models used for plasticity of polycrystalline metals, but with important conceptual differences. Firstly, to avoid model instability in post-peak softening, a kinematic constraint is used instead of a static one [34]. Thus, the strain (rather than stress) vector on each microplane is the projection of the macroscopic strain tensor. So we have,

$$
\epsilon_{N}=\epsilon_{i j} N_{i j} \epsilon_{M}=\epsilon_{i j} M_{i j} \epsilon_{L}=\epsilon_{i j} L_{i j}
$$

where $\epsilon_{N}, \epsilon_{M}$ and $\epsilon_{L}$ are the magnitudes of the three strain vectors corresponding to each microplane, and $N_{i j}=n_{i} n_{j}, M_{i j}=\left(n_{i} m_{j}+\right.$ $\left.m_{i} n_{j}\right) / 2$ and $L_{i j}=\left(n_{i} l_{j}+l_{i} n_{j}\right) / 2, n, m$, and $l$ being the three mutually orthogonal normal and tangential vectors characterizing that microplane, and the subscripts $i$ and $j=1,2,3$. Secondly, a variational principle (principle of virtual work) is used to relate the stresses on the microplanes $\left(\sigma_{N}, \sigma_{M}\right.$ and $\left.\sigma_{L}\right)$ to the macrocontinuum stress tensor $\sigma_{i j}$, to ensure equilibrium and is expressed as,

$$
\frac{2 \pi}{3} \sigma_{i j} \delta \epsilon_{i j}=\int_{\Omega} \sigma_{N} \delta \epsilon_{N}+\sigma_{M} \delta \epsilon_{M}+\sigma_{L} \delta \epsilon_{L} d \Omega
$$

This expression means that, within a unit sphere, the virtual works of macro-stresses and micro-stresses must be equal (for details, see [34, 36]). In the microplane model M7, the micro-stresses are subjected to strain dependent boundaries (or strength limits) of four types, viz.:

1. The tensile normal boundary-to capture progressive tensile fracturing;

2. The compressive volumetric boundaryto capture phenomena such as pore collapse under extreme pressures;

3. The compressive deviatoric boundaryto capture softening in compression; and

4. The shear boundary-to capture friction.

The M7 also includes the quasi-static rate effects [37], which consist of a rate-dependent crack opening and growth controlled by the activation energy of bond breakage, and of viscoelasticity of the material between the cracks. However, the quasi-static rate effects suffice only up the strain rate of about $1 / \mathrm{s}$, which is insufficient for impact.

Since comminution is induced by local shear strains, we assume that in a given strain increment $\mathrm{d} \epsilon_{i j}$, the additional energy to be dissipated must equal the additional distortional strain energy given by $\Delta \sigma_{D i j} \epsilon_{D i j}$ where $\Delta \sigma_{D i j}$ is the additional deviatoric stress that results due to scaled boundaries. To express it in terms of the microplane stresses, we first define the volumetric stress on the microplane level, $\sigma_{V}$, as

$$
\frac{2 \pi}{3} \frac{\sigma_{k k}}{3} \delta \epsilon_{m m}=\int_{\Omega} \sigma_{V} \delta \epsilon_{V} d \Omega
$$

Substituting $\delta \epsilon_{V}=\delta \epsilon_{m m} / 3$ and $\int_{\Omega}=2 \pi$, the volumetric stress on the microplane level can be expressed as $\sigma_{V}=\sigma_{k k} / 3$. Also, the microplane normal stresses $\sigma_{N}=\sigma_{D}+\sigma_{V}$ where $\sigma_{D}$ is the deviatoric stress on the microplane level. Likewise, for microplane strains, we have $\epsilon_{N}=\epsilon_{D}+\epsilon_{V}$. Note that $\epsilon_{V}=\epsilon_{k k} / 3$ is the same for all microplanes.

Then, subtracting Eq. (12) from (11) and 
noting that $\int_{\Omega} \sigma_{D} \delta \epsilon_{V}=\int_{\Omega} \sigma_{V} \delta \epsilon_{D}=0$, we get $\frac{2 \pi}{3} \sigma_{D i j} \delta \epsilon_{i j}=\int_{\Omega} \sigma_{D} \delta \epsilon_{D}+\sigma_{M} \delta \epsilon_{M}+\sigma_{L} \delta \epsilon_{L} d \Omega$

Then, since $\delta \epsilon_{D}=\delta \epsilon_{N}-\delta \epsilon_{V}=\delta \epsilon_{i j} N_{i j}-$ $\delta \epsilon_{i j} \delta_{i j} / 3, \delta \epsilon_{M}=\delta \epsilon_{i j} M_{i j}$ and $\delta \epsilon_{L}=\delta \epsilon_{i j} L_{i j}$, we get an expression for the macroscale deviatoric stress tensor as,

$$
\begin{aligned}
& \frac{2 \pi}{3} \sigma_{D i j}= \\
& \int_{\Omega} \sigma_{D}\left(N_{i j}-\frac{\delta_{i j}}{3}\right)+\sigma_{M} M_{i j}+\sigma_{L} L_{i j} d \Omega
\end{aligned}
$$

This expression implies that a change in the macro-scale deviatoric stress can be achieved by scaling only the deviatoric and frictional boundaries of M7. Let the change in the microplane deviatoric and shear stresses be $\Delta \sigma_{D}$, $\Delta \sigma_{M}$ and $\Delta \sigma_{L}$ for a change in deviatoric stress of $\Delta \sigma_{D i j}$. So we have,

$$
\begin{aligned}
& \frac{2 \pi}{3} \Delta \sigma_{D i j}= \\
& \int_{\Omega} \Delta \sigma_{D}\left(N_{i j}-\frac{\delta_{i} j}{3}\right)+\Delta \sigma_{M} M_{i j}+\Delta \sigma_{L} L_{i j} d \Omega
\end{aligned}
$$

Now, in the interest of simplicity, we assume that both the deviatoric and frictional boundaries are scaled by the same amount, on each microplane. So, $\Delta \sigma_{D}=\Delta \sigma_{M}=\Delta \sigma_{L}=\Delta f$, and

$$
\begin{aligned}
& \frac{2 \pi}{3} \Delta \sigma_{D i j}= \\
& \int_{\Omega} \Delta f\left(N_{i j}-\frac{\delta_{i j}}{3}\right)+\Delta f M_{i j}+\Delta f L_{i j} d \Omega
\end{aligned}
$$

This may be simplified as,

$$
\Delta \sigma_{D i j}=\Delta f C_{i j}
$$

where,

$$
C_{i j}=\frac{3}{2 \pi} \int_{\Omega}\left(N_{i j}-\frac{\delta_{i j}}{3}\right)+M_{i j}+L_{i j} d \Omega
$$

which is constant. $\Delta f$ may be taken out of the integral since we assume it to be the same for each microplane. Now, the next task is to relate the quantity $\Delta f$ to the energy dissipated due to comminution, $\Delta \mathcal{K}$. To ensure the aforementioned energy balance, the work of the additional deviatoric stress must dissipate an energy equal to the drop of kinetic energy of strain rate field caused by comminution in each increment. So,

$$
\mathrm{d}(-\Delta \mathcal{K})=\Delta \sigma_{D i j} \mathrm{~d} \epsilon_{D i j}
$$

where $i, j=1,2,3$ and denotes a small increment. So we have

$$
\mathrm{d}(-\Delta \mathcal{K})=\Delta f C_{i j} \mathrm{~d} \epsilon_{D i j}
$$

Next, using eq. 9 and taking the derivative with respect to time on both sides, we get

$$
\frac{2 A}{3} \dot{\epsilon}_{D}^{-1 / 3} \ddot{\epsilon}_{D}=\Delta f C_{i j} \dot{\epsilon}_{D i j}
$$

Multiplying both sides by $\dot{\epsilon}_{D i j}$, we obtain

$$
\frac{2 A}{3} \dot{\epsilon}_{D}^{-1 / 3} \ddot{\epsilon}_{D} \dot{\epsilon}_{D i j}=2 \Delta f C_{i j} \dot{\epsilon}_{D}^{2}
$$

since $\dot{\epsilon}_{D}=\sqrt{\dot{\epsilon}_{i j} \dot{\epsilon}_{i j} / 2}$. Thus we have,

$$
\Delta f C_{i j}=\frac{A}{3} \dot{\epsilon}_{D}^{-7 / 3} \ddot{\epsilon}_{D} \dot{\epsilon}_{D i j}
$$

Note that the right-hand side of this expression is consistent with [25]. To obtain the scalar value $\Delta f$, we now calculate the effective values of both sides, by taking square root of the inner product with itself. So we have,

$$
\Delta f\left(C_{i j} C_{i j}\right)^{1 / 2}=\frac{\sqrt{2} A}{3} \dot{\epsilon}_{D}^{-7 / 3} \ddot{\epsilon}_{D} \dot{\epsilon}_{D}
$$

Thus the scalar $\Delta f$ is expressed as,

$$
\Delta f=A_{1} \dot{\epsilon}_{D}^{-4 / 3} \ddot{\epsilon}_{D}
$$

Here $A_{1}$ is a constant to be calibrated, and is given by $A_{1}=\sqrt{2} A / 3 \bar{C}$ and $\bar{C}=\sqrt{C_{i j} C_{i j}}$. Therefore, the deviatoric and frictional boundaries of $\mathrm{M} 7$ are scaled as $F=F_{q s}(1+$ $\left.A_{1} \dot{\epsilon}_{D}^{-4 / 3} \ddot{\epsilon}_{D}\right)$, where $F_{q s}=F_{0}(1+h(\dot{\epsilon}))$, the 
boundary that is already scaled to account for the quasi-static rate effects [37], and $F_{0}$ is the original unscaled M7 boundary. This expression shows that, to capture the energy dissipation due to comminution, it is necessary to make the boundary a function of both the first and second time derivatives of the effective deviatoric strain.

\section{CONCRETE SLAB PERFORATION BY PROJECTILE IMPACT}

The M7 model with scaled boundaries was evaluated using the tests of projectile perforation, performed at the Geo-technical and Structure Laboratory of the US. Army Engineer Research and Development center (ERDC), Vicksburg [8, 38]. These tests used circular slabs of four thicknesses, 127, 216, $254 \mathrm{~mm}$ and 280 $\mathrm{mm}$, made of concrete WES-5000, whose standard compression strength was $48 \mathrm{MPa}$. The slabs were cast in steel culvert pipes of diameter $1.52 \mathrm{~m}$, sufficient to approximate the response for a semi-infinite radius (in spite of that, non-reflecting finite elements producing no backward waves were used at the boundary). The projectiles, which were hollow and made of steel, had an ogival-nose (caliber radius head 3.0, length/diameter ratio 7.0, and diameter 50.8 $\mathrm{mm}$ ) and weight of $2.3 \mathrm{~kg}$. The projectiles impacted the concrete slabs with the velocity of $310 \mathrm{~m} / \mathrm{s}$ at the angle of 90 degrees. The perforation tests were carried out two or three times for each thickness of the slab.

First, the M7 model was calibrated to fit the test data for concrete WES-5000, used in these tests. This concrete had Young's modulus $E=$ $25 \mathrm{GPa}$, and Poisson's ratio $\mu=0.18$. The optimum values of M7 parameters, which achieved very good fits of quasi-static uni-, bi- and triaxial tests, were $k_{1}=11 \times 10^{-5}, k_{2}=110, k_{3}=$ $30, k_{4}=100$ and $k_{5}=10^{-4}$ [34, 35].

The mesh used to discretize the slabs was random but statistically uniform, and consisted of tetrahedral elements of average size $7.5 \mathrm{~mm}$. The projectile was considered rigid since no obvious damage, melt or deformation was observed after the test. To prevent spurious mesh sensitivity, the modeling was performed in the sense of the crack band model, in which the finite element size (or mesh size) should be equal to the material characteristic length, which characterizes the size of the representative volume element (RVE) of the material and is used as the localization limiter. The element size was considered as $7.5 \mathrm{~mm}$, which is about 1-2 times the maximum aggregate size. Note that if, in quasi-static problems, the element size is changed, the crack band model requires adjusting the post-peak softening of the damage constitutive law so that the energy dissipated in the crack band (localized into one element width) would not change [39] (which is what is done in commercial software such as ATENA or OOFEM [40]). But, in projectile impact problems, the deformation is generally so fast that there is not enough time for the cracking damage to localize, and so the post-peak of the damage law need not, and should not, be adjusted.

Also note that, while the apparent strength and fracture energy depend on the strain rate, the crack band width itself does not. It is a material property that can be measured, e.g., as the minimum possible spacing of parallel quasistatic macro-cracks. It depends on material heterogeneity, and is usually equal to 1 to 3 maximum inhomogeneity sizes. In dynamic fracture, where the localization is suppressed by high deformation rates, the finite element solver based on the crack band model automatically simulates (in a diffuse way, of course) the formation of multiple cracks and crack branching. The additional energy dissipation at very high strain rates is accounted for by material law adjustment, one type of which is presented here.

For these analyses, it was necessary to remove excessively distorted elements to avoid termination of analysis due to negative Jacobian. This was done using an element deletion criterion based on the maximum principal strain. Thresholds of the criterion were set independently for tension and compression, to a value such that a further increase of the threshold did not make a difference to the predicted exit velocities. These values turned out to be 
$40 \%$ for tensile strain and $100 \%$ for compressive strain as shown in [27].

Next, using the aforementioned element deletion criterion, the quasi-static rate effects were employed, and the projectile exit velocities were predicted (with the same parameters as mentioned in [24]). These effects mainly refer to the rate dependence of cohesive crack opening and follow from the activation energy theory of bond ruptures. These effects are accounted for by scaling all the boundaries of M7 as $F_{q s}=F_{0}\left(1+C_{R 2} \ln \left(2 \dot{\epsilon} / C_{R 1}\right)\right)$ [37], where $\dot{\epsilon}=\sqrt{\frac{1}{2} \dot{\epsilon}_{i j} \dot{\epsilon}_{i j}}$. The parameter values $C_{R 1}$ and $C_{R 2}$ were determined in [24] to be $4 \times 10^{-6}$ and 0.022 respectively. The exit velocities were again predicted by including these effects, as shown in Fig. 3. It is seen that these effects caused the predicted velocities to change significantly. However, it is seen that the quasi-static rate effects still do not suffice to correctly predict the exit velocities. So, we now add the effects of comminution by scaling the deviatoric and friction boundaries of $\mathrm{M} 7$, as described earlier.

Parameter $A_{1}$ was calibrated by fitting one data point in Fig. 2, which led to the value $A_{1}=3 \times 10^{-7}$. The remaining points were then predicted. Fig. 2 shows the predicted exit velocities for all four slabs tested. As can be seen, the predictions of exit velocities are reasonably good, for all the data points. For the smaller slabs, the exit velocity is slightly overestimated while for the larger slab it is slightly underestimated. The fit could further be improved by relaxing the assumption that both the deviatoric and friction boundaries are scaled by the same factor. But this has not been explored since the errors are small and the data are too limited for calibrating two factors. To further assess the predictions, the predicted crater shapes for the slabs are compared to the measured ones, as shown in Fig. 3. It can be seen that the shapes can be matched very well, especially those at the exit side. This serves to show that the proposed scaling of M7 boundaries accounts for the phenomenon of comminution quite well.

\section{CONCLUSIONS}

1. The microplane model M7 with boundaries modified according to the kinetic energy theory of comminution is an effective approach to simulate projectile impact effects on concrete slabs and offers possibilities of further refinement. It can accurately predict projectile exit velocities, crater shapes and penetration depths.

2. It is necessary to scale up the deviatoric and friction boundaries of $\mathrm{M} 7$, to achieve a rate-dependent increase of the macroscopic deviatoric stress. Greater versatility could be obtained by scaling up the deviatoric and friction boundaries by different factors. But there are not enough test data to calibrate two independent factors.

3. The scaling of the boundaries (or strength limits) must be proportional to both: 1) the $-4 / 3$ power of the effective deviatoric strain rate, and 2) the time derivative of that rate.

4. The crack band model with a random tetrahedral mesh leads to accurate predictions of the crater shapes.

5. An element deletion threshold is necessary to run impact analyses. The choice of the threshold has a significant effect on the results. Chosen is high enough threshold for maximum principal strain such that a further increase would not change the results appreciably.

6. What is most important is that the finite element code dissipate the correct energy required by the kinetic theory of comminution. But how exactly this energy is dissipated does not seem important. Dissipation modes in terms of additional viscosity, rate dependence of interface fracture, and scaling of strength limits give similar results. While the viscosity approach is more natural for rate-dependent 
stress, the present approach has the advantage of greater versatility for further refinements, such as the possibility of different scalings of different microplane boundaries, which might be needed for various extensions such as hypervelocity impact, impact on rock, or explosive comminution of rock, e.g., shale.

Acknowledgments: Support under grant W911NF-15-1-0240 from the U.S. Army Research Office, Durham, to Northwestern University, is gratefully acknowledged.

\section{REFERENCES}

[1] Cadoni, E., Labibes, K., Albertini, C., Berra, M., Giangrasso, M., 2001. Strainrate effect on the tensile behaviour of concrete at different relative humidity levels. Materials and structures 34, 21-26.

[2] Camacho, G., Ortiz, M., 1996. Computational modelling of impact damage in brittle materials. International Journal of solids and structures 33, 2899-2938.

[3] Deshpande, V., Evans, A., 2008. Inelastic deformation and energy dissipation in ceramics: a mechanism-based constitutive model. Journal of the Mechanics and Physics of Solids 56, 3077-3100.

[4] Doyoyo, M., 2002. A theory of the densification-induced fragmentation in glasses and ceramics under dynamic compression. International journal of solids and structures 39, 1833-1843.

[5] Ferri, E., Deshpande, V., Evans, A., 2010. The dynamic strength of a representative double layer prismatic core: A combined experimental, numerical, and analytical assessment. Journal of Applied Mechanics 77,061011 .

[6] Forquin, P., Gary, G., Gatuingt, F., 2008. A testing technique for concrete under confinement at high rates of strain. International Journal of Impact Engineering $35,425-446$.
[7] Adley, M.D., Frank, A.O., Danielson, K.T., 2012. The high-rate brittle microplane concrete model: Part I: Bounding curves and quasi-static fit to material property data. Computers \& Concrete 9, 293-310.

[8] Frank, A.O., Adley, M.D., Danielson, K.T., McDevitt Jr, H.S., 2012. The highrate brittle microplane concrete model: Part II: application to projectile perforation of concrete slabs. Computers \& Concrete $9,311-325$.

[9] Gailly, B.A., Espinosa, H.D., 2002. Modelling of failure mode transition in ballistic penetration with a continuum model describing microcracking and flow of pulverized media. International journal for numerical methods in engineering 54, 365-398.

[10] Gatuingt, F., Pijaudier-Cabot, G., 2002. Coupled damage and plasticity modelling in transient dynamic analysis of concrete. International journal for numerical and analytical methods in geomechanics $26,1-$ 24.

[11] Grady, D., 1982. Local inertial effects in dynamic fragmentation. Journal of Applied Physics 53, 322-325.

[12] Grady, D., 1998. Shock-wave compression of brittle solids. Mechanics of Materials $29,181-203$.

[13] Grady, D., Kipp, M., 1979. The micromechanics of impact fracture of rock, International Journal of Rock Mechanics and Mining Sciences \& Geomechanics Abstracts. Elsevier, pp. 293-302.

[14] Grady, D., Kipp, M., 1995. Experimental measurement of dynamic failure and fragmentation properties of metals. International journal of solids and structures 32, 2779-2791. 
[15] Grady, D.E., 1990. Particle size statistics in dynamic fragmentation. Journal of applied physics 68, 6099-6105.

[16] Kožar, I., Ožbolt, J., 2010. Some aspects of load-rate sensitivity in visco-elastic microplane material model. Computers and Concrete 7(4), pp.317-329.

[17] Mescall, J., Weiss, V., 1984. Materials behavior under high stress and ultrahigh loading ratesPart II, Proceeding of 29th Sagamore Army Conference, Army Materials and Mechanics Center, Watertown, MA.

[18] Mott, N., 1947. Fragmentation of shell cases. Proceedings of the Royal Society of London. Series A. Mathematical and Physical Sciences 189, 300-308.

[19] Ožbolt, J., Sharma, A., Reinhardt, H.-W., 2011. Dynamic fracture of concrete compact tension specimen. International Journal of Solids and Structures 48, 15341543.

[20] Shih, C., Nesterenko, V., Meyers, M., 1998. High-strain-rate deformation and comminution of silicon carbide. Journal of applied physics 83, 4660-4671.

[21] Wei, Z., Evans, A., Deshpande, V., 2009. The influence of material properties and confinement on the dynamic penetration of alumina by hard spheres. Journal of Applied Mechanics 76, 051305.

[22] Bažant, Z.P., Caner, F.C., 2013. Comminution of solids caused by kinetic energy of high shear strain rate, with implications for impact, shock, and shale fracturing. Proceedings of the National Academy of Sciences 110, 19291-19294.

[23] Bažant, Z.P., Caner, F.C., 2014. Impact comminution of solids due to local kinetic energy of high shear strain rate: I. Continuum theory and turbulence analogy. Journal of the Mechanics and Physics of Solids 64, 223-235.

[24] Caner, F.C., Bažant, Z.P., 2014. Impact comminution of solids due to local kinetic energy of high shear strain rate: II. Microplane model and verification. Journal of the Mechanics and Physics of Solids 64, 236-248.

[25] Su, Y., Bažant, Z.P., Zhao, Y., Salviato. M., Kirane., K., 2015. Viscous energy dissipation of kinetic energy of particles comminuted by high-rate shearing in projectile penetration, with potential ramification to gas shale. Int. J. Fract. 51, pp 3142.

[26] Bažant, Z.P., Su, Y., 2015. Impact comminution of solids due to progressive crack growth driven by kinetic energy of high-rate shear. ASME J. of Applied Mechanics 82, 031007-1-031007-5.

[27] Kirane, K., Su, Yewang, Bažant, Z.P. (2015). Strain-rate-dependent microplane model for high-rate comminution of concrete under impact based on kinetic energy release theory. Proc. Royal Soc. A, 471 (2182), 20150535.

[28] Freund, L.B. (1990). Dynamic Fracture Mechanics. Cambridge University Press, Cambridge, U.K.

[29] Shockey, D. A., Curran, D. R., Seaman, L., Rosenberg, J. T., Petersen, C. F., 1974. Fragmentation of Rock under Dynamic Loads. Int. J. Rock Mech. Sci. and Geomech. Abstr. Vol. 11, pp. 303-317.

[30] Hemmert, D.J., Smirnov, V.I., Awal, R., Lati, S., Shetty, A., 2010. Pulsed power generated shockwaves in liquids from exploding wires and foils for industrial applications, Proceedings of the 16th International Symposium on High Current Electronics (Tomsk, Russia), pp. 537-540. 
[31] Maurel, O., Reess, T., Matallah, M., De Ferron, A., Chen, W., La Borderie, C., Pijaudier-Cabot, G., Jacques, A., Rey-Bethbeder, F., 2010. Electrohydraulic shock wave generation as a means to increase intrinsic permeability of mortar. Cement and Concrete Research 40, 16311638.

[32] Reinhardt, H.W., Weerheijm, J., 1991. Tensile fracture of concrete at high loading rates taking account of inertia and crack velocity effects. Int. J. Fract. 51, pp 31-42.

[33] Schuhmann Jr, R., 1940. Principles of Comminution, I-Size Distribution and Surface Calculations. AIME Technical Publication 1189.

[34] Caner, F.C., Bažant, Z.P., 2012a. Microplane model M7 for plain concrete. I: Formulation. Journal of Engineering Mechanics 139, 1714-1723.

[35] Caner, F.C., Bažant, Z.P., 2012b. Microplane model M7 for plain concrete. II: Calibration and verification. Journal of Engineering Mechanics 139, 1724-1735.

[36] Bažant, Z.P., Caner, F.C., Carol, I., Adley, M.D., Akers, S.A., 2000. Mi- croplane model M4 for concrete. I: Formulation with work-conjugate deviatoric stress. Journal of Engineering Mechanics 126, 944-953.

[37] Bažant, Z.P., Caner, F.C., Adley, M. D., Akers, S. A. 2000. Fracturing rate effect and creep in microplane model for dynamics. ASCE J. Eng. Mech. 126, pp 962-970.

[38] Cargile, J.D., 1999. Development of a constitutive model for numerical simulation of projectile penetration into brittle geomaterials. DTIC Document.

[39] Bažant, Z.P., and Oh, B.-H. 1983. Crack band theory for fracture of concrete. Materials and Structures (RILEM, Paris), 16, 155-177.

[40] Červenka, J., Bažant, Z.P., and Wierer, M. 2005. Equivalent localization element for crack band approach to mesh-sensitivity in microplane model. Int. J. for Numerical Methods in Engrg. 62 (5), 700-726.

[41] Bažant, Z.P., Adley, M. D., Carol, I., Jirasek, M., Akers, S. A., Rohani, B., Cargile, J. D., and Caner, F.C., 2000. Large strain generalization of microplane model for concrete and application. ASCE J. Eng. Mech. v. 126, no. 9, pp 971-980. 


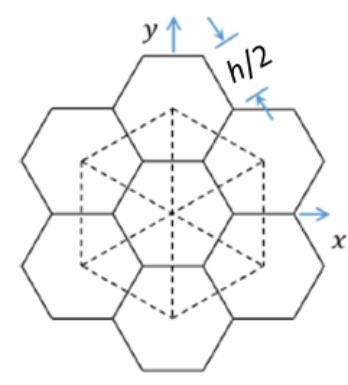

(a)

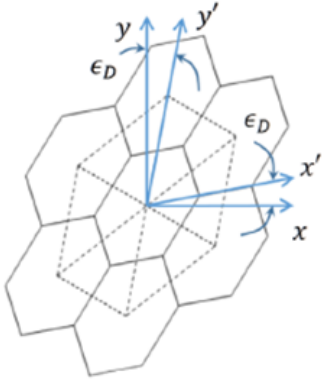

(b)

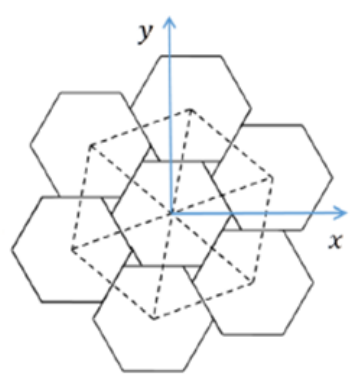

(c)

Figure 1: Schematic illustration of material comminution into prismatic hexagonal particles: (a) undeformed regime; (b) sheared regime; (c) comminuted regime.

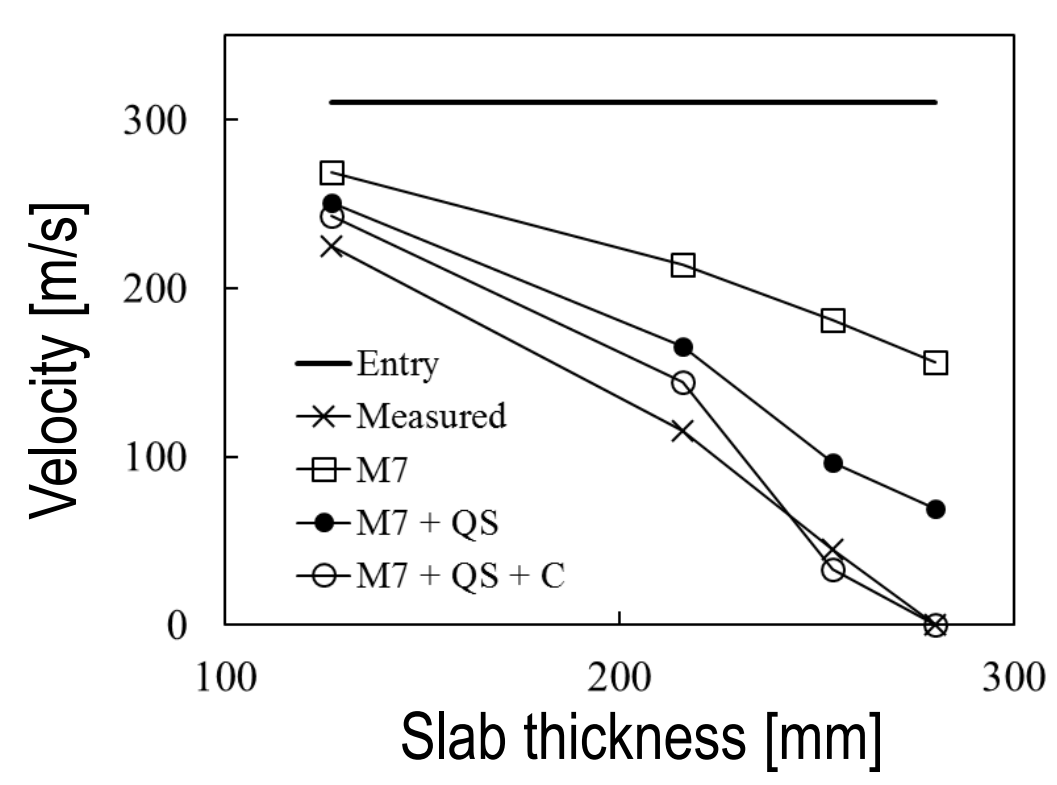

Figure 2: Comparison of the exit velocities predicted by M7 with no rate effects, with quasi-static rate effects and with comminution effects and the experimental data 

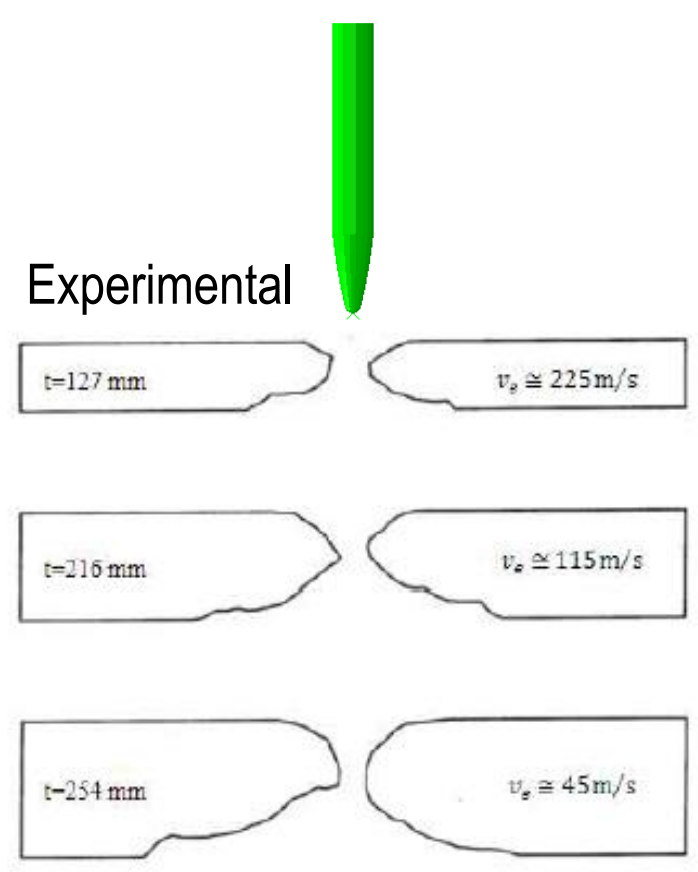

\section{Predicted}
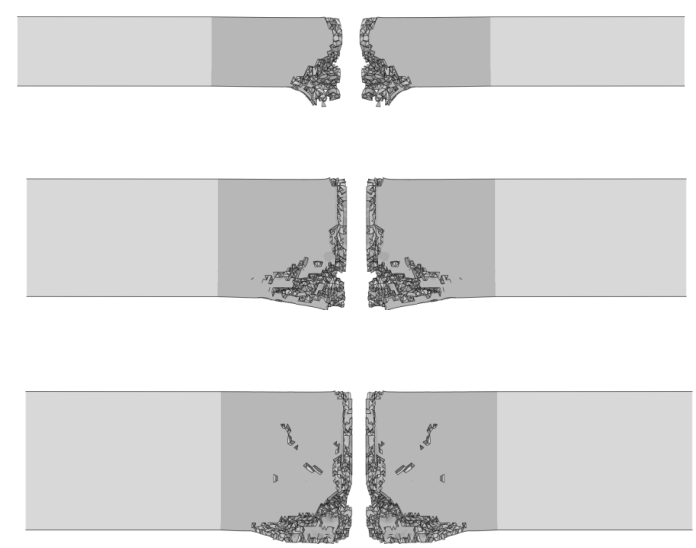

Figure 3: Comparison of the crater shapes predicted by M7 with comminution effects and the experiment data 